\title{
Hybrid plasmonic/photonic crystals for optical detection of bacterial contaminants
}

\author{
Giuseppe M. Paternò1*, Liliana Moscardi1,2, Stefano Donini1, Aaron M. Ross2, Silvia M. Pietralunga1,3, Nicholas Dalla \\ Vedova1, Simone Normani1, Ilka Kriegel4, Guglielmo Lanzani1,2 and Francesco Scotognella
}

\author{
1Center for Nano Science and Technology, Istituto Italiano di Tecnologia (IIT), Via Pascoli 10, 20133, Milano, Italy \\ 2Physics Department, Politecnico di Milano, Piazza L. da Vinci 32, 20133 Milano, Italy \\ 3Institute for Photonics and Nanotechnologies (IFN), Consiglio Nazionale delle Ricerche (CNR), Piazza L. da Vinci 32, \\ 20133 Milano, Italy \\ 4Department of Nanochemistry, Istituto Italiano di Tecnologia (IIT), via Morego, 30, 16163 Genova, Italy
}

\begin{abstract}
Here, we show that a hybrid plasmonic/photonic crystal consisting of a thin layer of bioactive plasmonic material (i.e. silver) deposited on top a $1 \mathrm{D} \mathrm{PhC} \mathrm{can} \mathrm{detect} \mathrm{one} \mathrm{of} \mathrm{the} \mathrm{most} \mathrm{common} \mathrm{bacterial}$ contaminant, namely Escherichia coli. We speculate that the change in the plasmon charge density brought about by metal/bacterium interaction results in a variation of the plasmon resonance that, in turns, translates in a shift of the photonic structural color.
\end{abstract}

\section{Introduction}

Photonic crystals (PhCs) have been largely employed as detection/sensing devices in recent years, since the photonic stop-band can be tuned by applying a number of external stimuli, such as chemical $^{1}$, thermal ${ }^{2}$ and mechanical triggers ${ }^{3}$. In this context, we have recently proposed porous 1D photonic structures exhibiting electro-optical tunability, due to the incorporation of optoelectronically-active plasmonic nanoparticles in the photonic structures. $^{4-6}$

Here, we show that a hybrid plasmonic/photonic crystal consisting of a thin layer of bioactive plasmonic material (i.e. silver) deposited on top a 1D PhC can detect one of the most common bacterial contaminant, namely Escherichia coli. ${ }^{7}$ We speculate that the change in the plasmon charge density brought about by metal/bacterium interaction results in a variation of the plasmon resonance which, in turns, translates in a shift of the photonic structural color.

\section{Results}

We integrated the bio-responsive silver layer on top of a 1D $\mathrm{PhC}$ consisting of alternating layers of $\mathrm{SiO}_{2}$ and $\mathrm{TiO}_{2}$ nanoparticles ( 5 bilayers). In this context, the plasmonic metal can be seen as defective layer of the photonic crystal that affects the optical response of the $\mathrm{PhC}$ via its free carrier density.> Thus, the main goal is to modify the dielectric properties at the $\mathrm{PhC} /$ metal interface and, thus, the overall optical read-out by exploiting the possible change in the silver complex dielectric function caused by $\mathrm{Ag} /$ bacteria interaction. To localise strongly the plasmonic response at the interface of the photonic structure, we selected the minimum Ag thickness attainable with our deposition apparatus. Interestingly, while interaction with the culture medium (pristine) does not alter appreciably the photonic read-out (Fig. 1), when the hybrid structure is interfaced with the bacterial colonies we noticed a $40 \mathrm{~nm}$ blue-shift of the photonic band-gap.

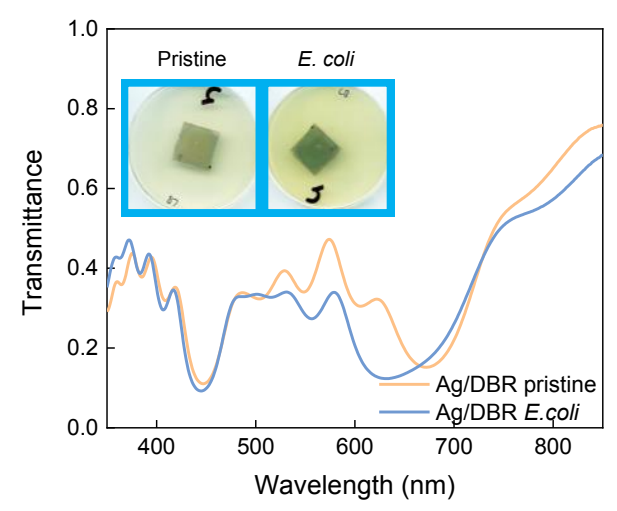

Fig. 1. Transmission spectrum of the pristine and E.colicontaminated Ag/DBR. The inset shows the visual change in the reflection properties of the sample.

\section{Conclusions}

Here, we show that a novel hybrid plasmonic-photonic device consisting of a thin layer of silver deposited on top of a solution-processed BS is responsive to bacterial contamination. In particular, we assessed the validity of our approach by detecting one of the most hazardous Gram-negative bacterial contaminants in food and water, Escherichia coli.

\section{References}

1. Hong, W., Hu, X., Zhao, B., Zhang, F. \& Zhang, D. 22, 5043-5047 (2010).

2. Kanai, T., Lee, D., Shum, H. C., Shah, R. K. \& Weitz, D. A. Adv. Mater. 22, 4998-5002 (2010).

3. Fudouzi, H. \& Sawada, T. Langmuir 22, 13651368 (2006).

\footnotetext{
* Corresponding author: giuseppe.paterno@iit.it
} 
4. Aluicio-Sarduy, E. et al. Beilstein J. Nanotechnol. 7, 1404-1410 (2016).

5. Paternò, G. M. et al. Sci. Rep. 8, 3517 (2018).

6. Paternò, G. M., Moscardi, L., Kriegel, I., Scotognella, F. \& Lanzani, G. J. Photonics Energy 8, 1 (2018).

7. Paternò, G. M. et al. J. Phys. Chem. Lett. 10, 4980-4986 (2019).

8. Drude, P. Ann. Phys. 312, 687-692 (1902). 